\title{
Influence value of deformation on the evolution of structure of monocrystal zirconium shear pressure
}

\author{
L.J. Egorova, Y.V. Khlebnikova†, V.P. Pilyugin \\ †Yulia_kh@imp.uran.ru
}

M.N. Miheev Institute of Metal Physics of Ural Branch of RAS, 18 Kovalevskaya St., Yekaterinburg, 620990, Russia

\begin{abstract}
The research of formation and the following evolution of $\omega$-phase in pseudo single crystal $\alpha$-zirconium during the deformation in Bridgman anvils at a pressure of $8 \mathrm{GPa}$, room temperature with the variety of the angles of rotation of the has been made by TEM. Has been discovered that phase $\alpha \rightarrow \omega$ transformation occurs not in the whole material, but in the areas of preexisting deformation twins. The initiation of development of processes of dynamic recrystallization by the deformation of zirconium to megaplastic values $(e \geq 5)$ under pressure has been detected. This is based on direct TEM-observations of regular polyhedra-shaped grains with varying degrees of defects of the internal microstructure. The effect of dynamic recrystallization in zirconium occurs due to the low level of thermal activity. The stabilization of structural elements of both $\alpha$ - and $\omega$-phases has been observed while reaching the deformation degree $e \geq 8$. At this stage of deformation speed of accumulation of defects in grain and movements of the growing grain is limited by the ratio of development of competing processes of fragmentation and dynamic recrystallization. Extremely deformed microstructure contains of misaligned to different angles microcrystallites average size $40-50 \mathrm{~nm}$ with visible edges. Microcrystallites of $\omega$-phase have lots of defects in their internal microstructure while crystallites of $\alpha$-phase do not contain any defects. The presence of orientation bonding of $\omega$ and $\alpha$ phases after megadeformation has been detected, which witness the realization of $\omega \rightarrow \alpha$ transformation.
\end{abstract}

Keywords: pseudo-single zirconium, megaplastic intense deformation, high hydrostatic pressure, $\alpha \rightarrow \omega$ phase transitions, recrystallization.

\section{Влияние величины деформации на эволюцию структуры} монокристаллического циркония при сдвиге под давлением

\author{
Егорова Л.Ю., Хлебникова Ю.В. ${ }^{\dagger}$, Пилюгин В.П. \\ †Yulia_kh@imp.uran.ru
}

Институт физики металлов им. М.Н. Михеева УрО РАН, ул. Ковалевской 18, Екатеринбург, 620990, Россия.

Методом просвечивающей электронной микроскопии проведено исследование образования и последующей эволюции $\omega$-фазы в псевдомонокристаллическом $\alpha$-цирконии при деформации на наковальнях Бриджмена при комнатной температуре с приложенным давлением до 8 ГПа и угловой скоростью $\omega=1,0$ об/мин, углы поворота наковальни варьировали от $\varphi=0^{\circ}$ (деформация осадкой) до $N=10$ полных оборотов. Обнаружено, что фазовое $\alpha \rightarrow \omega$ превращение происходит не во всем объеме материала, а в областях ранее появившихся деформационных двойников. В участках, где не происходит образование барической $\omega$-фазы, $\alpha$-фаза сохраняет практически монокристаллическое состояние с повышенной плотностью дислокаций и начальной стадией формирования ячеистой дислокационной структуры. Было выявлено, что деформация циркония до мегапластических величин $(e \geq 5)$ под давлением инициирует развитие процессов динамической рекристаллизации. Это основано на непосредственных ПЭМ наблюдениях зерен в форме правильных полиэдров с разной степенью дефектности внутренней микроструктуры. Проявление эффекта динамической рекристаллизации в цирконии объясняется низким значением тепловой активности. При достижении степени деформации $e \geq 8$ наблюдали стабилизацию структурных элементов, как $\alpha$ - так и $\omega$-фазы. На этом этапе деформации скорость накопления дефектов в теле зерна и движения границы растущего зерна лимитируется соотношением развития взаимоконкурирующих процессов - фрагментации и динамической рекристаллизации. Предельно деформированная сформированная микроструктура состоит из разориентированных на большие углы микрокристаллитов среднего размера 40 - 50 нм с выраженными гранями. Микрокристаллиты $\omega$-фазы имеют более дефектную внутреннюю микроструктуру в отличие от кристаллитов $\alpha$-фазы, не содержащих дефектов. Обнаружено наличие ориентационной связи $\omega$ и $\alpha$ фаз после мегадеформации, что свидетельствует о реализации обратного $\omega \rightarrow \alpha$ превращения.

Ключевые слова: псевдомонокристалл циркония, интенсивная мегапластическая деформация, высокое квазигидростатическое давление, $\alpha \rightarrow \omega$ фазовые переходы, рекристаллизация. 


\section{1. Введение}

Развитие современных методов получения и аттестации объемных наноматериалов, в том числе интенсивная мегапластическая деформация (ИМПД), стимулирует интерес к задачам по исследованию метастабильных фаз, образующихся в металлах и сплавах под воздействием высокого давления, в частности в титане и цирконии. При этом действие пластической деформации приводит к дефектному кристаллическому состоянию, характеризуемому особым наномасштабным уровнем структурных составляющих, когда существенная доля атомов находится в состоянии отсутствия дальнего кристаллического порядка. Подобное структурное состояние отличается особыми физико-механическими свойствами, измененным гистерезисом фазовых переходов, увеличением прочности, изменением ресурса пластичности, характера разрушения и т.д.

Известно, что цирконий может существовать в трех различных кристаллических модификациях: $\beta, \alpha$ и $\omega$. Высокотемпературная $\beta$-фаза имеет объемноцентрированную кубическую решетку ( $a=3,568 \AA)$, тогда как низкотемпературная $\alpha$-фаза - гексагональную плотноупакованную решетку с соотношением параметров $c / a=1,593$ ( $a=3,231 \AA, c=5,146 \AA)$, несколько меньшим, чем для идеальной упаковки $(c / a=1,633)$, в условиях воздействия высокого давления из $\alpha$-фазы образуется $\omega$-фаза [1], которая имеет гексагональную (ГП) решетку с параметрами: $a=5,053 \AA, c=3,091 \AA$ $(c / a=0,612)$.

Наиболее полная информация по метастабильной барической $\omega$-фазе в Zr до недавнего времени была собрана в работе [2]. Ряд работ посвящен исследованию зависимости механических свойств Тi и $\mathrm{Zr}$ от присутствия в структуре метастабильной $\omega$-фазы $[3,4]$. В работах [5, $6,7]$ были рассмотрены кристаллографические аспекты полиморфного $\alpha \rightarrow \omega$ превращения в $\mathrm{Zr}$ и $\mathrm{Ti}$.

На сегодняшний день детально не выяснен вопрос о структурных этапах образования и эволюции $\omega$-фазы в переходных металлах при сдвиге под давлением. Кроме того, на данный момент большинство экспериментов были выполнены с использованием поликристаллических объектов, но монокристаллы этих металлов интересны тем, что их использование исключает влияние исходных границ зерен на картину эволюции деформируемой структуры.

\section{2. Цель работы}

В настоящей работе проведено исследование образования и последующей эволюции $\omega$-фазы в псевдомонокристаллическом $\alpha$-цирконии в процессе деформации сдвигом под давлением на наковальнях Бриджмена при комнатной температуре, с целью установления размерно-морфологических характеристик образующейся структуры и возможности протекания процессов динамической рекристаллизации при мегапластической деформации.

\section{3. Материал и методика эксперимента}

Для решения поставленных задач методом бестигельной электронно-лучевой зонной плавки были получены образцы исходного иодидного монокристалла $\alpha$-циркония чистотой 99,9\%. Пластическую деформацию дисковых образцов осуществляли в твердосплавных наковальнях Бриджмена при давлении 8 ГПа с угловой скоростью $\omega=1,0$ об/мин, углы поворота наковальни варьировали от $\varphi=0^{\circ}$ (деформация осадкой) до $N=10$ полных оборотов. Степень истинной деформации (e) рассчитывали на середине радиуса образца по формуле [8]:

$$
e_{\text {ист }}=\frac{2}{\sqrt{3}} \ln \left(1+\left(\frac{\varphi \cdot r}{h_{\text {кон }}}\right)^{2}\right)^{1 / 2}+\ln \frac{h_{0}}{h_{\text {кон }}} \text {. }
$$

Структурно-фазовое состояние образцов циркония после деформации исследовали электронно-микроскопически на просвет тонких фольг в микроскопах JEM-200CX и FEI CM-30.

\section{4. Результаты эксперимента и их обсуждение}

Исследуемый в данной работе цирконий в исходном состоянии имел псевдомонокристаллическое структурное состояние, полученное при $\beta \rightarrow \alpha$-полиморфном превращении в процессе зонной плавки [9]. Отметим, что в условиях зонного переплава происходит очистка металла от примесей, поэтому выращенный монокристалл содержал не более $0,02 \%$ примесей. Электронно-микроскопические исследования позволили определить, что в результате полиморфного превращения образовались пакеты $\alpha$-реек шести возможных вариантов кристаллографической ориентации, при соблюдении ориентационных соотношений Бюргерса $\{110\}_{\beta}||\{0001\}_{\alpha}$. В пределах одного пакета псевдомонокристалла разориентировка между отдельными рейками не превышала $1^{\circ}$. Внутри реек наблюдалась развитая дислокационная субструктура (рис. 1). Размеры $\alpha$-реек в исходном состоянии достигали - 15-60 мкм, внутри реек наблюдали субструктуру реечного типа с размерами 0,5-0,7 мкм.

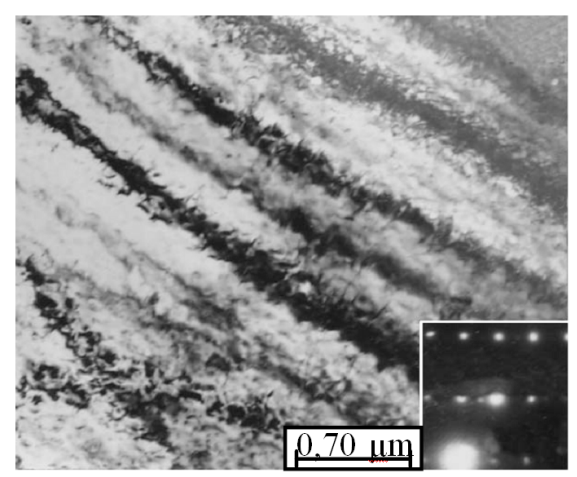

Рис. 1. Структура $\alpha$-реек исходного псевдомонокристалла $\mathrm{Zr}$. На дифракционной вкладке решетка с осью зоны [100] . [9]

Fig. 1. Structure of the $\alpha$-laths of the original pseudo-single $\mathrm{Zr}$. On the tab, the diffraction lattice with the axis zone $[100]_{\alpha}$. [9] 
Структурные исследования после деформации на наковальнях Бриджмена показали, что в процессе осадки $\varphi=0^{\circ}(e=0,2)$ при комнатной температуре в образце исходно псевдомонокристаллического циркония наблюдали в основном реечную структуру, частично двойникованную. В отдельных участках фиксировали наличие барической $\omega$-фазы, сохранившейся после снятия нагрузки.

Размеры субреек приблизительно соответствуют исходному состоянию, их ширина меняется от 0,1 мкм до 1 мкм. Электронно-микроскопические исследования позволили установить, что при такой деформации в крупных субрейках (от 0,6 до 1 мкм) $\alpha$-фаза сохраняет практически монокристаллическое состояние, соответствующее исходному - на полученных с данного участка электронограммах присутствуют рефлексы принадлежащие осям зон: [100] ${ }_{\alpha}$ и [010] ${ }_{\alpha}$ (рис. 2). Рефлексы $\omega$-фазы на электронограммах с таких участков не наблюдали.

В тех же областях, где после деформации осадкой, наблюдается появление барической $\omega$-фазы на электронномикроскопических снимках можно видеть, что прошло двойникование. Размер реек, в которых прошло фазовое превращение, варьируется от 0,03 до 0,2 мкм (рис. 3). Превращение $\alpha \rightarrow \omega$ проходит не во всем объеме, а лишь в тех рейках, ориентация которых наиболее благоприятна для выполнения условий протекания $\alpha \rightarrow \omega$ превращения. В работе [10] было определено, что вероятность фазового превращения при сжатии в чистом цирконии увеличивается, когда приложенное давление перпендикулярно оси $c$. В нашем случае на микродифракции, полученной с данной области, присутствуют отражения от участков $\omega$-фазы с осью зоны $[010]_{\omega}$, от кристаллитов $\alpha$-фазы с осью зоны $[13 \overline{1}]_{\alpha м}$, и двойника $\alpha$-фазы $[131]_{\alpha д}$, плоскость двойникования $(113)_{\alpha}$, при этом след плоскости двойникования проходит через плоскость $(5 \overline{2} \overline{1})_{\alpha}$. Вероятно, вследствие того, что исходный образец циркония имел псевдомонокристаллическую структуру, в процессе деформации осадкой было задействовано ограниченное количество кристаллографических плоскостей, что и привело к тому, что двойникование прошло по наименее активно реализуемой плоскости двойникования (113) циркония. Двойники по такой плоскости обнаруживаются либо после значительной деформации, либо перед разрушением $[11,12]$. Выполняются ориентационные соотношения (101) $\|$ (101) ${ }_{\omega}[5]$. Из рис. 3 видно, что двойники располагаются параллельно направлениям $[5 \overline{2} \overline{1}]_{\alpha} \|[001]_{\omega}$. Направление $[001]_{\omega}$ соответствует направлению, в котором залегают плоские дефекты в $\omega$-фазе $[5,13]$, а это может означать, что стимулом $\alpha \rightarrow \omega$ превращения, возможно, является сонаправленность плоских дефектов $\omega$-фазы и плоскости двойникования, в нашем случае $[5 \overline{2} \overline{1}]_{\alpha}$.

Реечная двойникованная субструктура наблюдается вплоть до $N=3$ об. (e $\approx 8)$ (рис. 4 ), на электронограммах от таких областей были обнаружены лишь единичные рефлексы $\omega$-фазы, присутствуют рефлексы $\alpha$-фазы двух ориентировок $[001]_{\alpha 1}$ и [011 $]_{\alpha 2}$. Направление $[001]_{\omega}$ здесь так же как при деформации осадкой совпадает с направлением двойников $\alpha$-фазы (рефлекс (001) „указан стрел-

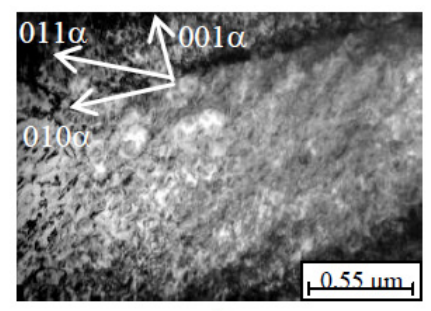

a
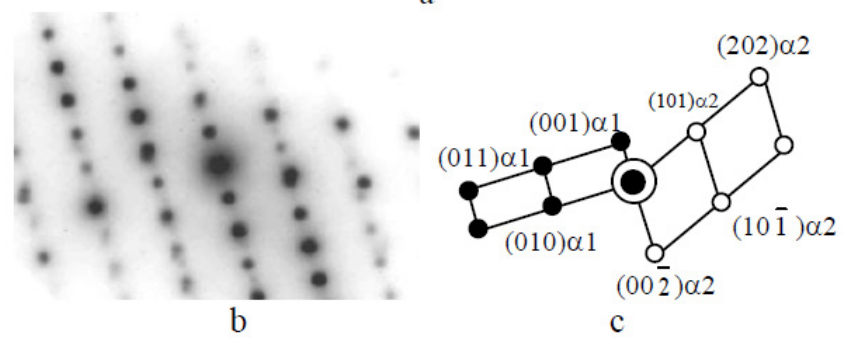

Рис. 2. Структура циркония после деформации осадкой: (a) светлопольное изображение; (b) дифракция с двух крупных кристаллитов; (c) - расшифровка электронограммы. Обратные решетки с осью зоны $[100]_{\alpha 1}$ и $[010]_{\alpha 2}$.

Fig. 2. Zirconium structure after deformation upsetting: (a) brightfield image; (b) diffraction with two large crystallites; (c) - decoding of electron diffraction. Reciprocal lattice with $[100]_{\alpha 1}$ zone axis and $[010]_{\alpha 2}$.
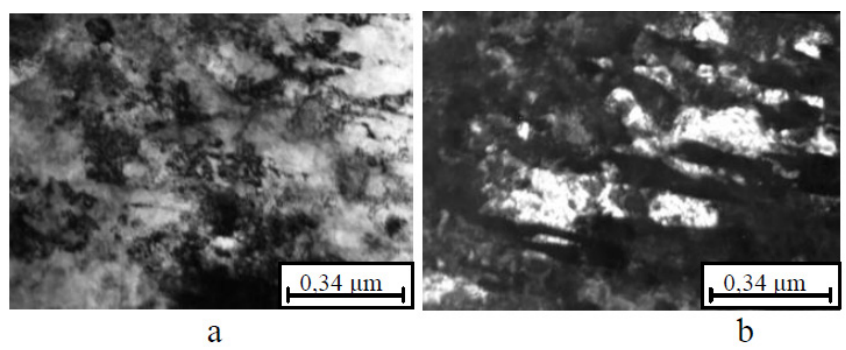

a

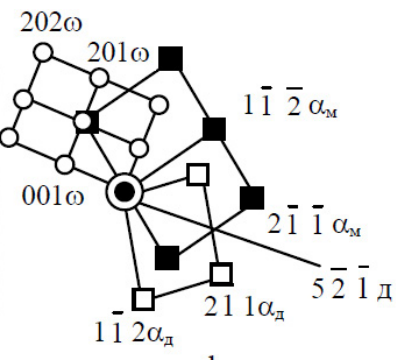

$\mathrm{d}$

Рис. 3. Структура циркония после деформации осадкой: (a) светлопольное изображение; (b) темнопольное изображение

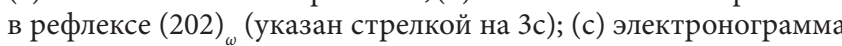
от исследуемого участка; (d) расшифровка электронограммы. Ось зоны обратной решетки $\omega$-фазы $[010]_{\omega}$, ось зоны матрицы $\alpha$-фазы $[13 \overline{1}]_{\alpha м}$, двойника $\alpha$-фазы $[131]_{\alpha д}$ след плоскости двойникования $(5 \overline{2} \overline{1})_{\alpha}$.

Fig. 3. Zirconium structure after deformation upsetting: (a) bright-field image; (b) dark-field image at reflex (202) (indicated by the arrow in 3c); (c) electron diffraction from the study area; (d) decoding of electron diffraction. The axis zone of the reciprocal lattice $\omega$-phase $[010]_{\omega}, \alpha$-phase matrix axis zone $[13 \overline{1}]_{\alpha \mathrm{m}}$, twin $\alpha$-phase $[131]_{\alpha \mathrm{d}}$, trace of the twinning plane $(5 \overline{2} \overline{1})_{\alpha}$. 
кой на рис. 4c). Тот факт, что при сохранении реечной структуры присутствие $\omega$-фазы минимально, можно объяснить двумя процессами: либо в процессе разгрузки после деформации реализуется обратное $\omega \rightarrow \alpha$ превращение, либо барическое $\alpha \rightarrow \omega$ превращение не происходит вовсе. Явление обратного фазового перехода в данных условиях более вероятно вследствие большого влияния межзеренных и межфазных границ. Т.е. обратное $\omega \rightarrow \alpha$ превращение становится возможным при большом соотношении длины межфазной границы к объему структурного элемента, что описывается известным соотношением Холла-Петча: $H=H_{0}\left(1+k d^{-1 / 2}\right)$.

В тех же участках деформированного на $N=3$ об. циркония, где рефлексы барической $\omega$-фазы образуют обратную решетку, наблюдается смешанная, по большей части мелкозернистая структура (рис. 5a), образованная вследствие фрагментации в процессе деформации. В таких областях присутствуют кристаллиты с четкой огранкой (рис. 5b), а так же структурные элементы с размытыми, насыщенными дефектами, границами (рис. 5c). Некоторые из кристаллитов имеют форму правильных полиэдров с углами в вершинах, близкими к $120^{\circ}$ с дефектной внутренней структурой (см. рис.5а во вкладке). Размер элементов, у которых четко прослеживаются

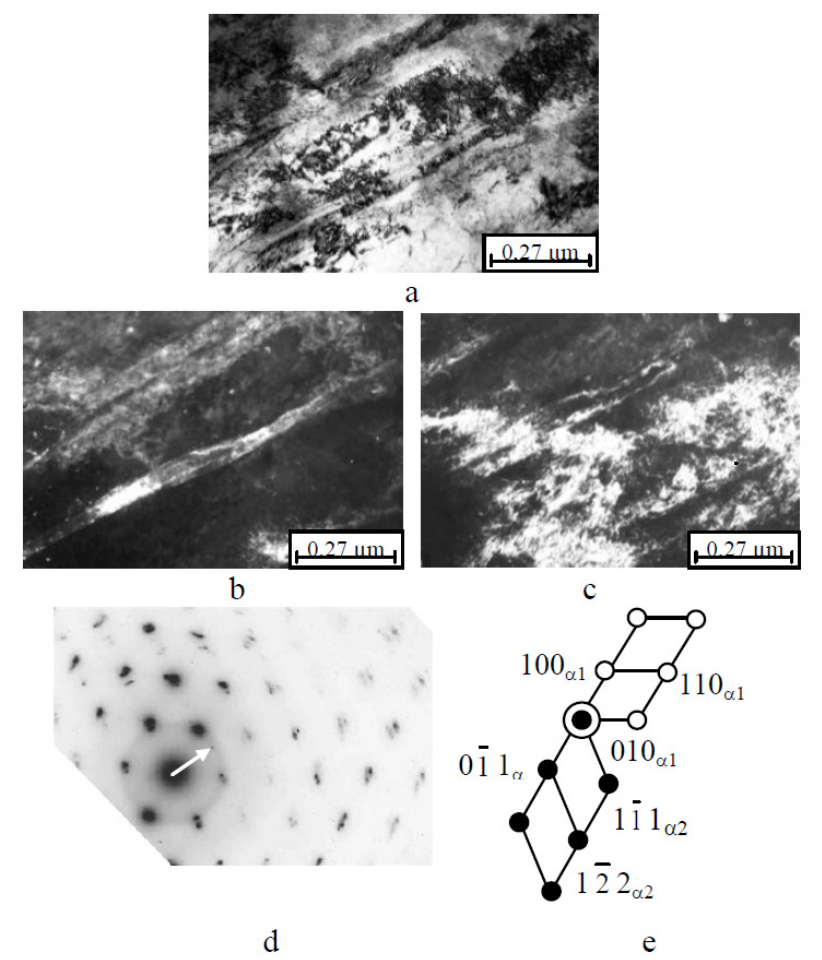

Pис. 4. Электронно-микроскопическое изображение структуры псевдомонокристаллического циркония, деформированного на $N=3$ об. $(e=8)$ : (а) светлопольное изображение; (b) темнопольное изображение в рефлексе (110) $)_{11}$; (c) темнопольное изображение в рефлексе (0ㄹㄹ) ${ }_{\alpha 2}$; (d) электроннограмма от исследуемого участка; (е) схема расшифровки. Обратные решетки с осью зоны $[001]_{\alpha 1}$ и $[011]_{\alpha 2}$.

Fig. 4. Electron-microscopic image of the structure pseudo-single $\mathrm{Zr}$ strain on $N=3$ vol. ( $(e=8)$ : (a) bright-field image; (b) darkfield image at reflex $(110)_{\alpha 1}$; (c) dark-field image at reflex $(0 \overline{2} 2)_{\alpha 2}$; (d) electron diffraction from the study area; (e) decoding of electron diffraction. The axis zone of the reciprocal lattice $[001]_{\alpha 1}$ and $[011]_{\alpha 2}$. границы, варьируется от 20 нм до 100 нм. На электронограмме с такого участка наблюдаются рефлексы, принадлежащие трем ориентировкам $\omega$-фазы: $[001]_{\omega 1},[1 \overline{1} 0]_{\omega 2}$ и $[010]_{\omega 3}$ (рис. $\left.5 \mathrm{~d}, \mathrm{e}\right)$. Совпадений по известным ориентационным соотношениям между $\alpha$ - и $\omega$-фазами не наблюдается.

Наличие четких структурных границ микрокристаллитов (рис. 5b), их размер и форма, а так же наличие внутренних дефектов может свидетельствовать о прошедшей в процессе деформации под давлением динамической рекристаллизации в цирконии, чего не наблюдалось в других $d$-переходных металлах (Fe, $\mathrm{Mo}, \mathrm{Ni}, \mathrm{Cr}$ ) при аналогичных режимах деформирования [14]. В титане явление динамической рекристаллизации впервые наблюдали при деформации $e>6$ при комнатной температуре [15]. В цирконии развитие динамической рекристаллизации, вероятно, как и в титане, стало возможным благодаря невысокой тепловой активности данного материала, что поспособствовало аккумуляции тепловой энергии материала и явилось, в нашем случае, причиной появления в деформированном цирконии локальных объемов, испытавших динамическую рекристаллизацию. Данная характеристика отвечает именно динамическим тепловым процессами и рассчитывается

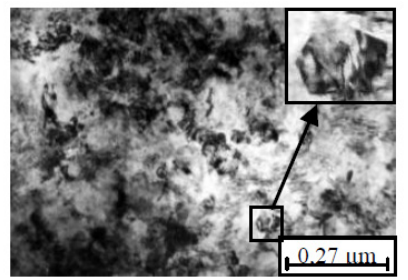

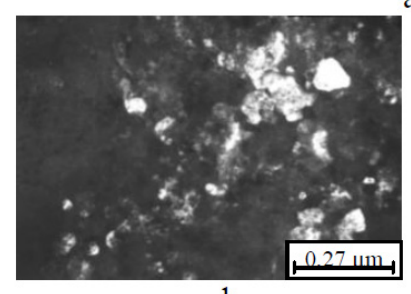

b

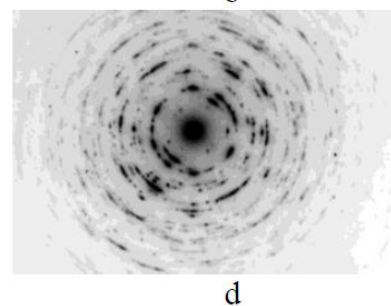

d

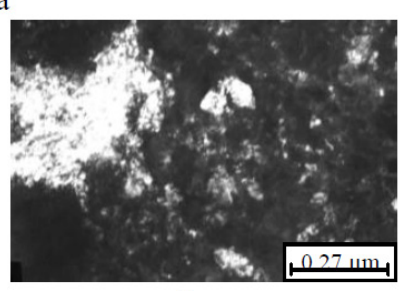

c

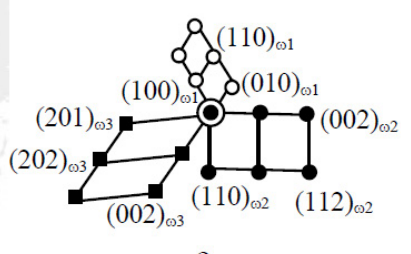

e
Рис. 5. Электронно-микроскопическое изображение структуры деформированного на $N=3$ об. $(e=8)$ псевдомонокристаллического циркония: (а) светлопольное изображение; (b) темнопольное изображение в рефлексе (110) (c) - темнопольное изображение в рефлексе (201) ${ }_{\omega 3}$; (d) электроннограмма от исследуемого участка; (e) схема расшифровки. Обратные решетки с осью зоны $[001]_{\omega 1},[1 \overline{1} 0]_{\omega 2}$ и $[010]_{\omega 3}$.

Fig. 5. Electron-microscopic image of the structure pseudo-single Zr strain on $N=3$ vol. ( $e=8)$ : (a) bright-field image; (b) darkfield image at reflex $(110)_{\omega 1}$; (c) dark-field image at reflex $(201)_{\omega 3}$; (d) electron diffraction from the study area; (d) decoding of electron diffraction. The axis zone of the reciprocal lattice $[001]_{\omega 1},[1 \overline{1} 0]_{\omega 2}$ and $[010]_{\omega 3}$. 
по формуле:

$$
e=\sqrt{\lambda \times \rho \times C}
$$

где $\lambda-$ теплопроводность материала, $\rho \times C-$ объемная теплоемкость [16].

С увеличением количества оборотов при сдвиге под давлением до $N=5(e>8)$ мы наблюдали уменьшение среднего размера структурных элементов циркония до 10 - 100 нм. Т.е. продолжаются процессы фрагментации и измельчения структуры. Как и при деформации на $N=3$ об., отдельные кристаллиты имеют четкие границы (рис. 6а), а некоторые не имеют четкой огранки, их границы насыщены дефектами. Реечная структура не наблюдается. Исходя из анализа полученных электронограмм можно сделать вывод, что количество $\alpha$-фазы значительно уменьшилось - присутствуют по большей части рефлексы от $\omega$-фазы. Наблюдаются отдельные подросшие зерна $\omega$-фазы размером 150 - 200 нм (рис. 6а). Они имеют четкую огранку, внутри зерен наблюдаются дефекты присущие $\omega$-фазе, залегающие параллельно направлению [001] . Между $\alpha$ - и $\omega$-фазами выполняются ориентационные соотношения [100] $\|_{\omega}[001]_{\alpha}$ (см. рис. 6е).

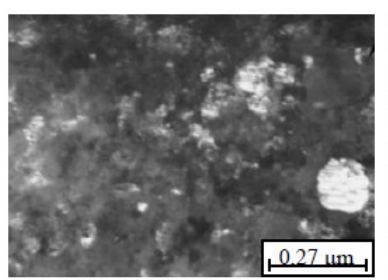

a

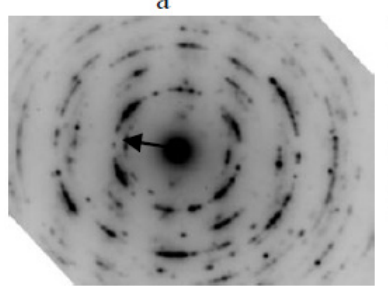

c

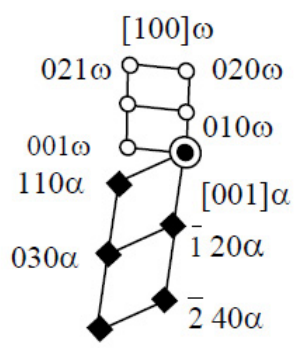

e

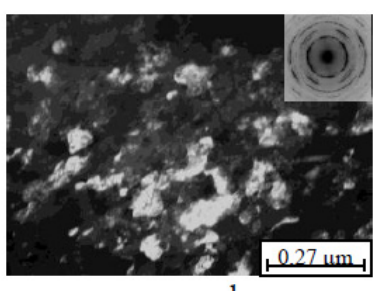

b

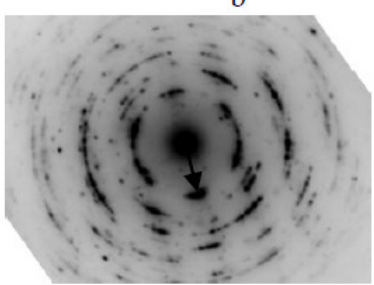

d

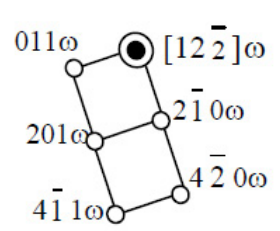

f

На электронограмме полученной с большой диафрагмой (рис. 6b во вкладке) четко прослеживаются текстурные максимумы (утолщения) в направлениях $[201]_{\omega},[011]_{\omega}$ и $[210]_{\omega}$, рефлексы данных направлений принадлежат к оси зоны $\omega$-фазы [122] (рис. 6f). Отсюда можно предположить, что, несмотря на то, что часть деформированной структуры рекристаллизовалась, текстура деформации еще сохранилась.

Увеличение степени деформации $(N=10$ об., $e \approx 10)$ практически не приводит к изменению среднего размера субмикрокристаллической структуры (10-180 нм), то есть фрагментация при дальнейшей деформации оказалась энергетически не выгодным процессом. Структура после мегадеформации однородна и изотропна, наблюдается большое количество ограненных микрокристаллитов с размерами от 20 до 50 нм, часть из которых относится к $\omega$-фазе (рис. 7). Между $\alpha$ - и $\omega$-фазой наблюдаются такие же ориентационные соотношения, как при деформации на $N=5$ об. [100] $\|_{\omega}[001]_{\alpha}$. Наличие ориентационной связи после мегадеформации может свидетельствовать о реализации обратного $\omega \rightarrow \alpha$ превращения. Обнаруживаемая $\omega$-фаза сохраняет направленность внутренних дефектов, они располагаются параллельно [001] $($ рис. 7b, d). Это может послужить еще одним свидетельством того, что стимулом к образованию $\omega$-фазы является появление плоских дефектов, или сдвиг в направлении $[100]_{\alpha} \|[001]_{\omega}$ [4]. Мегадеформация не меняет структуру $\omega$ фазы. Известно [4], что барическая фаза имеет бо́льшую твердость и плотность, она более устойчива к деформации, чем $\alpha$-фаза циркония. Кроме того, сохранение внутренней структуры барической фазы, подвергнутой большим деформациям, может быть свидетельством происходящей, в процессе деформирования, рекристаллизации. Текстурные

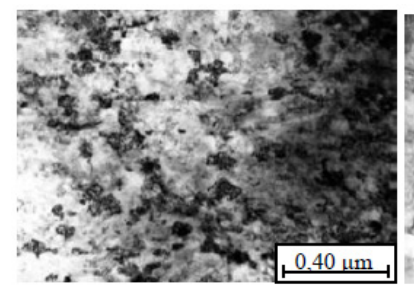

a

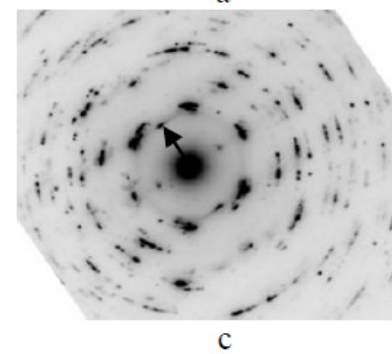

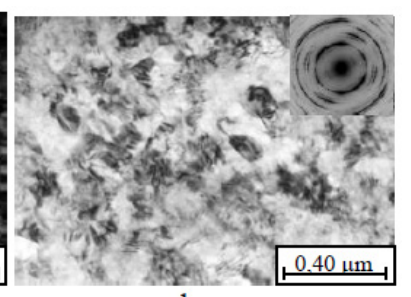

$\mathrm{b}$

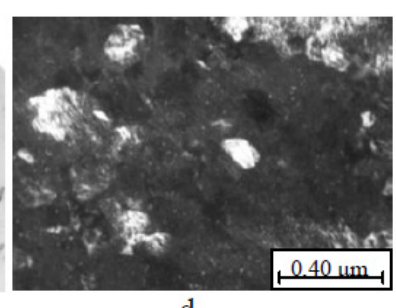

Рис. 7. Электронно-микроскопическое изображение структуры деформированного на $N=10$ об. $(e=10)$ псевдомонокристаллического циркония: $(\mathrm{a}, \mathrm{b})$ светлопольные изображения; (c) электронограмма от исследуемого участка; (d) темнопольное изображение в рефлексе $(001)_{\omega}$ (показан стрелкой на рис. 7c).

Fig. 7. Electron-microscopic image of the structure pseudo-single $\mathrm{Zr}$ strain on $N=10$ vol. $(e=10)$ : (a, b) bright-field image; (c) electron diffraction from the study area; (d) dark-field image at reflex $(001)_{\omega}$ (indicated by the arrow in $7 \mathrm{c}$ ). 
максимумы на электронограмме, снятой с большой диафрагмой, наблюдаются в тех же направлениях, что и у деформированного на $N=5$ об. образца циркония: $[201]_{\omega},[011]_{\omega}$ и $[210]_{\omega}$, принадлежащие оси зоны $\omega$-фазы [122] (рис. 7b).

\section{5. Заключение}

Методом просвечивающей электронной микроскопии обнаружено, что при деформации осадкой на наковальнях Бриджмена псевдомонокристалла циркония с приложенным давлением до 8 ГПа фазовое $\alpha \rightarrow \omega$ превращение происходит не во всем объеме материала, а только в областях ранее появившихся деформационных двойников. При этом, преимущественными местами образования новой фазы будут области ориентированные таким образом, что плоскостям двойникования $\alpha$-фазы соответствуют плоскости залегания планарных дефектов в образующейся $\omega$-фазе.

В участках, где, в процессе деформацией осадкой, не происходит образование барической $\omega$-фазы, $\alpha$-фаза сохраняет практически монокристаллическое состояние с повышенной плотностью дислокаций и начальной стадией формирования ячеистой дислокационной структуры.

Обнаружено, что деформация циркония до мегапластических величин $(e \geq 5)$ под давлением инициирует развитие процессов динамической рекристаллизации. Это основано на непосредственных ПЭМ наблюдениях зерен в форме правильных полиэдров с разной степенью дефектности внутренней микроструктуры. Проявление эффекта динамической рекристаллизации при мегадеформации в цирконии объясняется низким значением его тепловой активности.

При достижении степени деформации $e \geq 8$ наблюдали стабилизацию структурных элементов, как $\alpha$ - так и $\omega$-фазы. На этом этапе деформации скорость накопления дефектов в теле зерна и движения границы растущего зерна лимитируется соотношением развития взаимоконкурирующих процессов фрагментации и динамической рекристаллизации. Предельно деформированная сформированная микроструктура состоит из разориентированных на большие углы микрокристаллитов среднего размера 40-50 нм с выраженными гранями. Микрокристаллиты $\omega$-фазы имеют более дефектную внутреннюю микроструктуру в отличие от кристаллитов $\alpha$-фазы, не содержащих дефектов.

Обнаружено наличие ориентационной связи между $\omega$ - и $\alpha$-фазами циркония после мегадеформации, что может свидетельствовать о реализации обратного $\omega \rightarrow \alpha$ превращения.

Благодарность/Aknowledgements. Электронно-микроскопическое исследование деформированных образиов титана проводили на микроскопах JЕM-200СХ и СM-30 Philips в отделе электронной микроскопии ЦКП "Испьтательный иентр нанотехнологий и перспективных материалов" Института физики металлов УрО РАН. Работа выполнена в рамках государственного задания ФАНО России по теме “Структура", № 01201463331.

\section{Литература/References}

1. Jemieson J. C. Science, 1963, v. 140, p. 72

2. Tonkov E.Yu. Phase diagrams of elements at high pressure. M.: Science, Home edition of Physical and mathematical literature, 1979. $192 \mathrm{p}$ (in Russian) [Тонков Е. Ю. Фазовые диаграммы элементов при высоком давлении. М.: Наука, Главная редакция физико-математической литературы, 1979. 192 с.]

3. Hongxiang Zong, Dezhen Xue, Xiangdong Ding and Turab Lookman. Journal of Physics: Conference Series. 2014. V.500. P. 112042

4. Cerreta E.K., Escobedo J.P., Rigg P.A., Trujillo C.P., Brown D.W., Sisneros T.A., Clausen B., Lopez M.F., Lookman T., Bronkhorst C.A., Addessio F.L.. Acta Materialia. 2013. V. 61. P. 7712 - 7719

5. Alshevsky Y.L., Kulnitsky B.A., Konyaev Y.S., Osipov M.P. FMM 1984, t.58, vol. 4, pp. 795-803 (in Russian) [Альшевский Ю.Л., Кульницкий Б.А., Коняев Ю.С., Усиков М.П. ФММ, 1984, т.58, вып. 4, стр. 795-803]

6. Dobromyslov A.V., Taluts N.I., Demchuk K.M., Martem'yanov A.N. FMM 1988 m. 65, no. 3, pp. 588 - 593 (In Russian) [Добромыслов А. В., Талуц Н. И., Демчук К.М., Мартемьянов А.Н. ФММ 1988, т. 65, вып. 3, стр. 588 - 593]

7. X. Shen, P.F. Yu, Q. Jing, Y. Yao, L. Gu, Y.G. Wang, X.F. Duan, R.C. Yu, and R.P. Liu. Scripta Materialia 67 (2012) 653-656

8. M. V. Degtyarev, T.I. Chashchukhina, L. M. Voronova, L.S. Davydova and V.P. Pilyugin. The Physics of Metals and Metallography. Vol. 90, No. 6, 2000 p. 604-612.

9. Yu. V. Khlebnikova, V.A. Sazonova, D.P. Rodionov, N.F. Vil'danova, L. Yu. Egorova, Yu. V. Kaletina, I. L. Solodova, and V.M. Umova. The Physics of Metals and Metallography. Vol. 108, No. 3, 2009 p. 254-263

10. B. Srinivasarao, A.P. Zhilyaev, M.T. Pérez-Prado. Orientation dependency of the alpha to omega plus beta transformation in commercially pure zirconium by highpressure torsion. Scripta Mater. Vol. 65, 2011. No. 3, p. $241-244$.

11. Rapperport E. J., Hartley C. S. “Trans. AIME”, v. 218, p. $869-877,1960$.

12. Papirov I.I., Tihinsky G.F. Nature zirconium plastic deformation. Kharkiv, KhPTI Ukrainian Academy of Sciences, 1976, 36 p (in Russian) [Папиров И.И., Тихинский Г.Ф. Природа пластической деформации циркония. Харьков, ХФТИ АН УССР, 1976, 36 стр.]

13. A. V. Dobromyslov, N. I. Taluts. 1990, № 5, 108-115 (In Russian) [А. В. Добромыслов, Н. И. Талуц. ФММ, 1990, № 5, c. 108 - 115]

14. A.M. Glezer and L.S. Metlov. Physics of the Solid State Vol. 52, No. 6, 2010 p. 1162 - 1169.

15. V.P. Pilyugin, Yu.V. Khlebnikova, L.Yu. Egorova, T.R. Suaridze, N.N. Resnina, and A.M. Patselov. The Physics of Metals and Metallography. Vol. 116, No. 12, 2015 p. $1203-1213$.

16. A. V. Lykov The theory of heat conduction. M.: "High School", 1967. 600 p. (In Russian) [Лыков А. В. Теория теплопроводности. М.: “Высшая школа”, 1967. 600 с.] 